09.2;09.5;09.7

\title{
Регистрация однофотонного сигнала от низколетящих спутников для целей спутникового квантового распределения ключей
}

\author{
() А.В. Хмелев ${ }^{1-3}$, А.В. Дуплинский ${ }^{3,4}$, В.Ф. Майборода ${ }^{3}$, Р.М. Бахшалиев ${ }^{3}$, М.Ю. Баланов ${ }^{3,5}$, \\ В.Л. Курочкин ${ }^{1,3,5, \text { Ф Ю.В. Курочкин }}{ }^{1-3,5}$ \\ ${ }^{1}$ Российский квантовый центр, Москва, Россия \\ ${ }^{2}$ Московский физико-технический институт (Национальный исследовательский университет), \\ Долгопрудный, Московская обл., Россия \\ ${ }^{3}$ КуРэйт, Москва, Россия \\ ${ }^{4}$ Московский государственный технический университет им. Н.Э. Баумана, Москва, Россия \\ ${ }^{5}$ Центр компетенций НТИ „Квантовые коммуникации“, Национальный исследовательский \\ технологический университет МИСиС, Москва, Россия \\ I E-mail: v.kurochkin@rqc.ru
}

Поступило в Редакцию 8 апреля 2021 г.

В окончательной редакции 28 мая 2021 г.

Принято к публикации 31 мая 2021 г.

\begin{abstract}
Продемонстрирована наземная приемная станция для квантового распределения ключа со спутником, способная анализировать квантовые поляризационные состояния фотонов. В эксперименте по сопровождению спутника низкой околоземной орбиты остаточная ошибка слежения наземной станцией не превышает $10 \mu \mathrm{rad}$, что обеспечивает стабильный прием оптического сигнала в четырех каналах декодера поляризационных состояний. Проведена оценка эффективности регистрации оптического сигнала в режиме сопровождения спутников.
\end{abstract}

Ключевые слова: квантовое распределение ключа, квантовые коммуникации, детектор одиночных фотонов, искусственный спутник Земли.

DOI: 10.21883/PJTF.2021.17.51387.18817

Квантовое распределение ключей (КРК) - это принципиально отличная от классической методика защиты информации, в которой случайная последовательность битов, т. е. ключ шифрования формируется между пользователями при помощи квантовых состояний передаваемых частиц. В качестве этих частиц удобно использовать одиночные фотоны, а кодировать передаваемую информацию при помощи неортогонального набора квантовых состояний фазы, поляризации или временно́го интервала этих частиц. Так, фотонные состояния передаются либо по оптоволокну $[1,2]$, но с ограничениями на расстояние, либо по открытому пространству, где основные потери вызваны дифракционной расходимостью оптического излучения и турбулентностью в атмосфере. Вместе с этим эксперименты в открытом пространстве проводятся как вдоль земной поверхности с использованием телескопов [3], дронов [4], так и между искусственным спутником Земли (ИСЗ) и наземными станциями $[5,6]$.

Цель настоящей работы заключается в проведении эксперимента по одновременному сопровождению спутника на низкой околоземной орбите и регистрации оптического сигнала в однофотонном режиме на основе разработанной наземной приемной станции для КРК со спутником.

Приемная станция системы спутникового КРК представлена на рис. 1 и состоит из зеркального телескопа (RCT) с фокусным расстоянием $4.8 \mathrm{~m}$, оптического декодера фотонных состояний (PA) [5,6], блока анализа и обработки оптических сигналов (APS) и блока электроники.

Вспомогательный телескоп-рефрактор (GT) с апертурой $70 \mathrm{~mm}$ и матрица камеры (САМ2) имеют поле зрения $0.7^{\circ} \times 0.7^{\circ}$ и реализуют для монтировки телескопа обратную оптическую связь в процессе сопровождения космических объектов. В результате этого вместе с контуром точной подстройки, а именно быстрым поворотным зеркалом (FSM) и высокоскоростной камерой (CAM1), формируется двухступенчатая система стабилизации оптического излучения в приемной станции [7]. Задача этой системы состоит в наведении телескопа на целевой объект, отслеживании и удержании оптического сигнала объекта на торце оптического волокна диаметром $105 \mu \mathrm{m}$.

Помимо регистрации однофотонных сигналов на длине волны $850 \mathrm{~nm}$ в соответствии с протоколом ВB84 в системе предусмотрен прием когерентных импульсов на длине волны $532 \mathrm{~nm} \mathrm{с} \mathrm{целью} \mathrm{синхронизации} \mathrm{однофо-}$ тонных состояний во времени, как это было сделано в работе [5]. Также эти когерентные импульсы используются как опорный оптический сигнал для контура точной подстройки, что позволяет получить необходимую точность стабилизации при удержании потока фотонов на торце оптического волокна.

Разделение квантовых состояний в стандартном оптическом декодере происходит по протоколу ВВ84, где реализован пассивный выбор базиса на основе равнопле- 


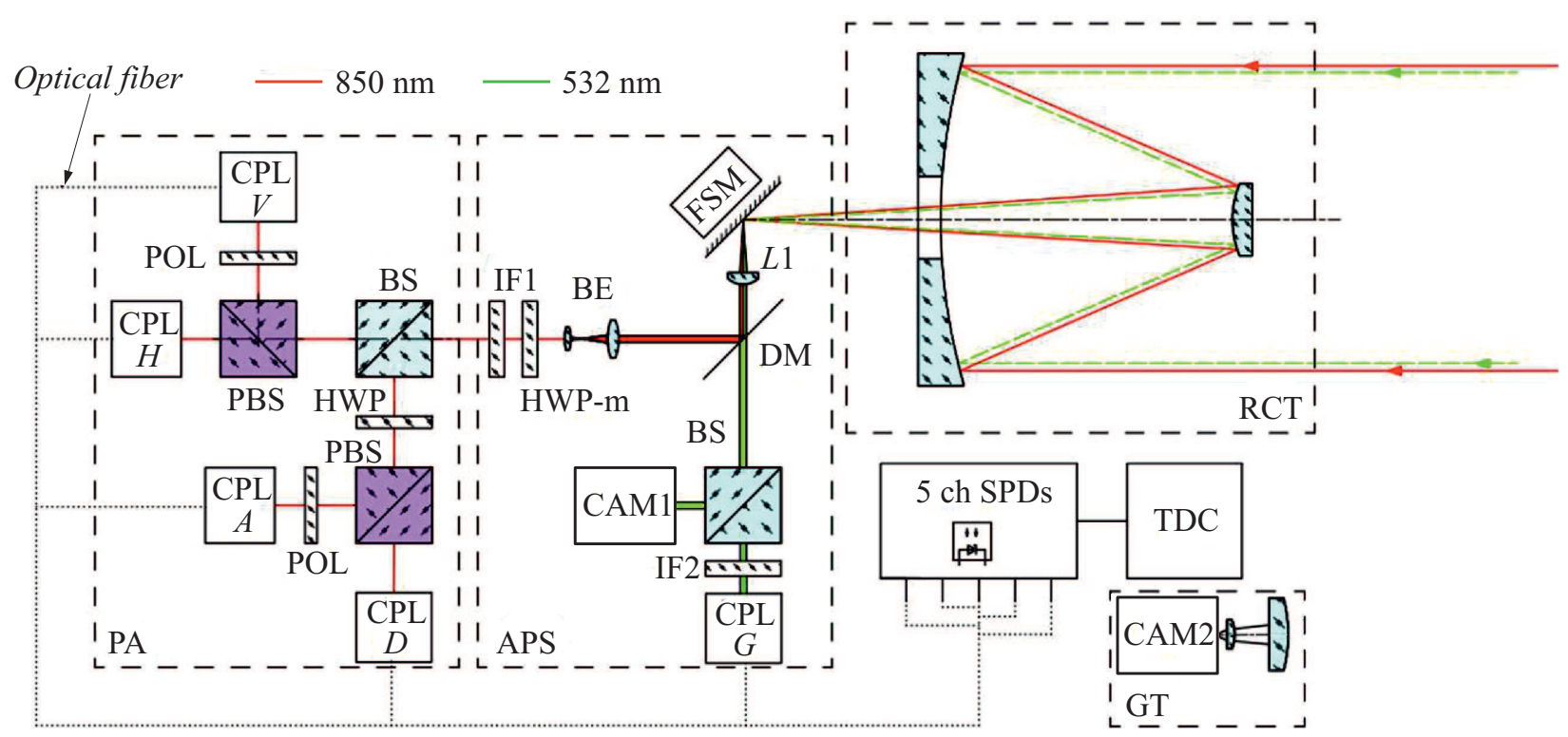

Рис. 1. Схема установки наземной станции. RCT - телескоп системы Ричи-Кретьена с апертурой $600 \mathrm{~mm}$; GT - вспомогательный телескоп; CAM2 - камера вспомогательного телескопа; APS - модуль анализа и обработки сигналов; FSM быстрое управляемое зеркало; DM - дихроичное зеркало; $L 1$ - линза $(f=75 \mathrm{~mm})$; $\mathrm{BS}$ - 50:50 светоделительный куб; CAM1 - камера (поле зрения $4 \times 4 \mathrm{mrad}, 100 \mathrm{fps}$ ); ВE - расширитель пучка; HWP-m - моторизованная полуволновая пластина; IF1 - интерференционный фильтр (центральная длина волны $850 \mathrm{~nm}$, полуширина $10 \mathrm{~nm}$ ); IF2 - интерференционный фильтр (центральная длина волны $532 \mathrm{~nm}$, полуширина $10 \mathrm{~nm}$ ); CPL - коллиматор; PA - поляризационный анализатор; PBS поляризационный светоделительный куб; POL - поляризатор; HWP - полуволновая пластина; optical fiber - oптическое волокно; 5 ch SPDs - пятиканальный модуль детекторов одиночных фотонов; TDC — время-цифровой преобразователь.

чего светоделительного куба (BS) [5,6]. Вслед за этим в каждом базисе располагается поляризационный светоделительный куб (PBS), который разделяет ортогональные поляризационные состояния на два оптических канала. В результате поляризационный коэффициент экстинкции, измеренный для всей приемной станции и усредненный по всем четырем каналам, составляет 250:1.

Следует отметить, что мы ожидаем относительное вращение ИСЗ и приемного модуля вокруг их общей оптической оси в процессе сеанса КРК. По этой причине предусмотрено использование моторизованной полуволновой пластинки (HWP-m) дополнительно к оптическому декодеру BВ84 с целью активной компенсации поворота базисов поляризации.

В результате фотоны, попавшие в оптическое волокно, преобразуются одним из пяти детекторов Excelitas Technologies SPCM-AQRH-14-FC (5 ch SPDs) в электрические импульсы, которым ставится в соответствие время их регистрации на время-цифровом преобразователе Swabian Instruments Time Tagger 20 (TDC).

Объектом исследования в работе был выбран видимый пролет искусственного спутника ERBS (Earth radiation budget satellite) на низкой околоземной орбите с высотой $405-417 \mathrm{~km}$. Время наблюдения и стабильного удержания освещенного Солнцем спутника приемной станцией составило $180 \mathrm{~s}$. В течение этого времени двухступенчатая система стабилизации отслеживала оптическое изображение объекта со среднеквадратичной угловой ошибкой менее $10 \mu \mathrm{rad}$. На рис. 2 показана зависимость угловой ошибки от времени, полученная при отклонениях центра изображения ERBS от координат целевой точки на матрице камеры (CAM1) с фокусным расстоянием объектива $25 \mathrm{~mm}$ и размером пиксела $5.5 \mu \mathrm{m}$.

В свою очередь удержание излучения от спутника на торце оптического волокна позволило промоделировать процесс регистрации квантовых состояний наземным модулем и оценить эффективность приема потока фотонов в режиме сопровождения спутников. Так, координаты целевой точки в эксперименте соответствовали наилучшему попаданию излучения в оптические волокна, а расчетная величина углового отклонения объекта от целевой точки, при которой сигнал в оптическом волокне пропадает, равнялась $30 \mu \mathrm{rad}$.

На рис. 3 представлены результаты измерений фотоотсчетов для четырех оптических каналов декодера в зависимости от времени слежения за спутником ERBS, проводимых в спектральном диапазоне $840-860 \mathrm{~nm}$. Центральная область наблюдения за ИСЗ характеризуется наибольшим уровнем счета, что обусловлено максимально возможным сближением спутника с наземной станцией. Также на рис. 3 наблюдаются кратковременные прерывания при регистрации оптического сигнала детекторами одиночных фотонов. Это вызвано превышением допустимой ошибки в $30 \mu \mathrm{rad}$ при отклонениях изображения ИСЗ от целевой точки на камере (рис. 2) 


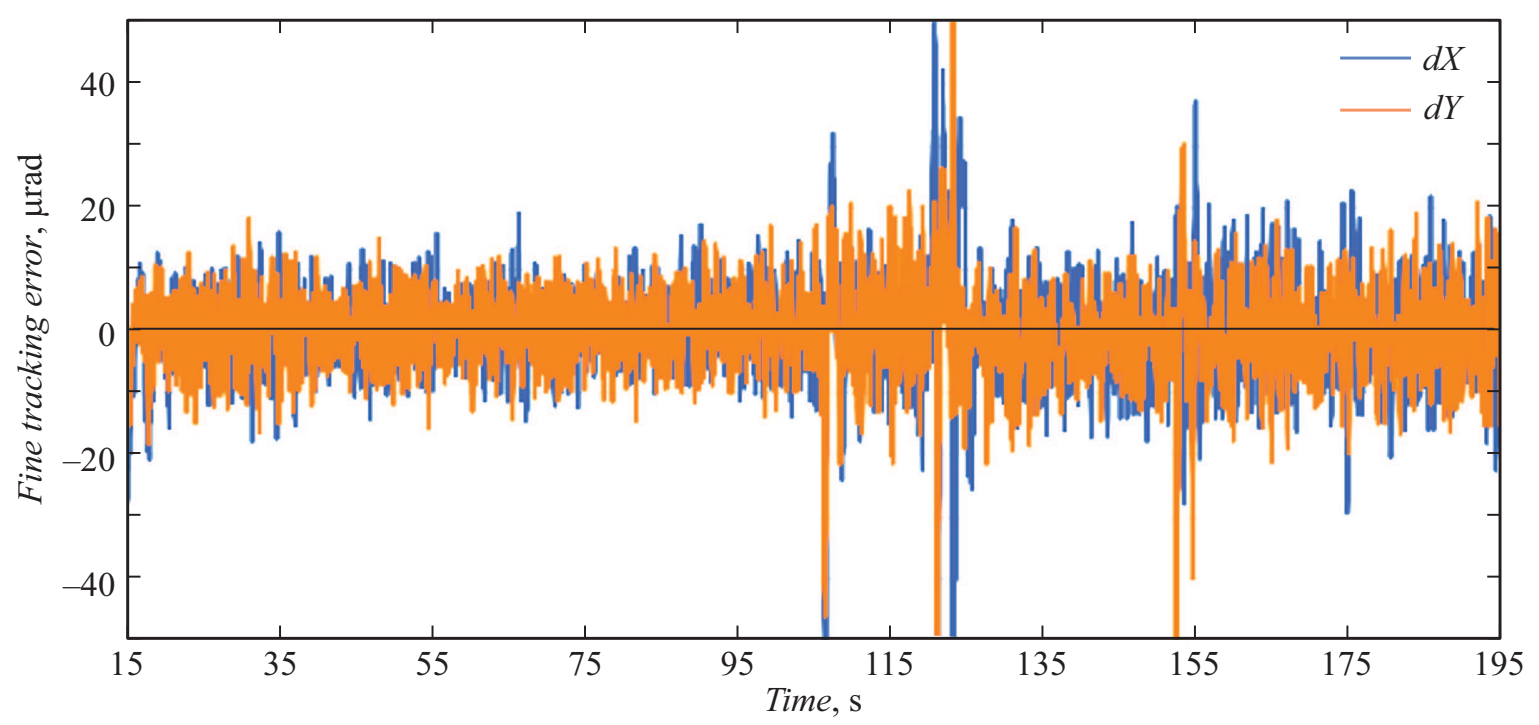

Рис. 2. Угловое отклонение изображения ERBS от целевой точки на матрице высокоскоростной камеры $(\mathrm{CAM} 1)$. $d X-$ отклонение по оси $X ; d Y$ - отклонение по оси $Y$.

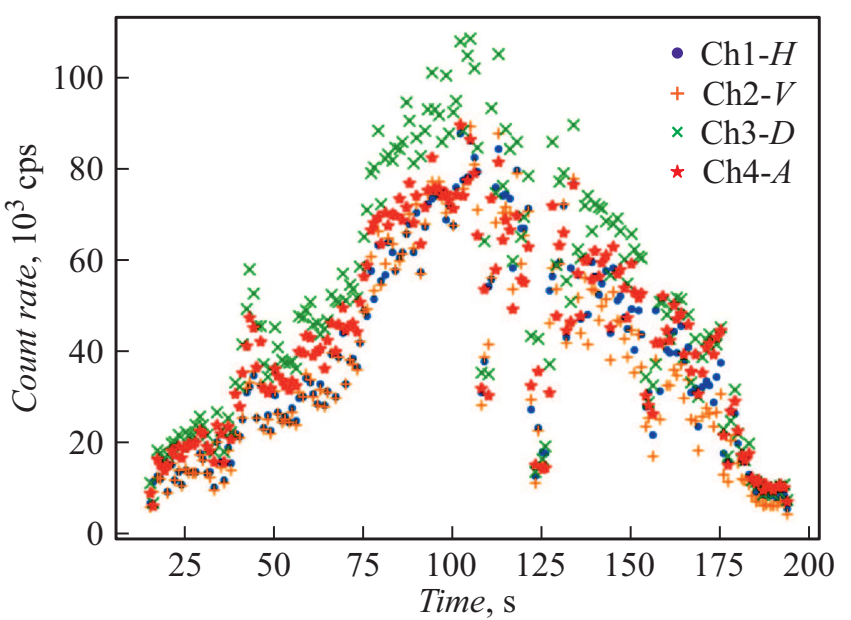

Рис. 3. Количество фотоотсчетов в секунду по четырем квантовым каналам от спутника ERBS. Ch - канал измерения состояний фотонов: $H-$ с горизонтальной $\left(0^{\circ}\right)$ поляризацией, $V-$ с вертикальной $\left(90^{\circ}\right)$ поляризацией, $D-$ с диагональной $\left(45^{\circ}\right)$ поляризацией, $A-\mathrm{c}$ антидиагональной $\left(-45^{\circ}\right)$ поляризацией.

и, как следствие, непопаданием в оптическое волокно отраженного от спутника солнечного света.

Звездная величина спутника ERBS в видимом диапазоне во время его пролета над наземной станцией достигала значения 2.20 согласно данным открытых источников по мониторингу ИСЗ. Такому блеску спутника можно сопоставить с некоторой точностью звездную величину со значением $1.60 \pm 0.15$ в спектральном диапазоне I фотометрической системы. Кроме того, при пролете спутника его суммарное значение фотоотсчетов по четырем каналам в максимуме достигало $350000 \mathrm{cps}$. Так, с целью определения уровня оптического сигнала от спутника в оптоволокне нами было проведено измерение фотоотсчетов от звезды Каф ( $\beta$ Cas) со схожей звездной величиной, равной 1.77 в спектральном классе I. Суммарное значение фотоотсчетов по четырем каналам составило $430000 \pm 80000 \mathrm{cps}$, где ошибка вызвана турбулентностью в атмосфере.

Таким образом, уровень оптического сигнала от спутника ERBS можно оценить, пересчитав его фотоотсчеты к звездной величине 1.77 и сравнив их с сигналом, регистрируемым от звезды Каф. Отсюда получается, что приведенное значение фотоотсчетов равно $300000 \pm 40000 \mathrm{fps}$, а уровень сигнала в сравнении со звездой равен $70 \pm 22 \%$.

В результате проведенных экспериментальных исследований показано, что среднеквадратичная ошибка удержания оптического изображения ИСЗ на низкой околоземной орбите не превышает $10 \mu \mathrm{rad}$ бо́льшую часть времени наблюдения, что обеспечивает стабильный прием оптического сигнала всеми каналами анализатора состояний. Полученное системой стабилизации значение ошибки и эффективность регистрации оптического сигнала демонстрируют готовность наземной станции к приему квантового ключа со спутников на низкой околоземной орбите.

\section{Финансирование работы}

Работа выполнена при поддержке Российского научного фонда по проекту № 17-71-20146.

\section{Конфликт интересов}

Авторы заявляют, что у них нет конфликта интересов. 


\section{Список литературы}

[1] J.P. Chen, C. Zhang, Y. Liu, C. Jiang, W. Zhang, X.L. Hu, J.Y. Guan, Z.W. Yu, H. Xu, J. Lin, M.J. Li, H. Chen, H. Li, L. You, Z. Wang, X.B. Wang, Q. Zhang, J.W. Pan, Phys. Rev. Lett., 124 (7), 070501 (2020). DOI: 10.1103/PhysRevLett.124.070501

[2] Y.A. Chen, Q. Zhang, T.Y. Chen, W.Q. Cai, S.K. Liao, J. Zhang, K. Chen, J. Yin, J.G. Ren, Z. Chen, S.L. Han, Q. Yu, K. Liang, F. Zhou, X. Yuan, M.S. Zhao, T.Y. Wang, X. Jiang, L. Zhang, W.Y. Liu, Y. Li, Q. Shen, Y. Cao, C.Y. Lu, R. Shu, J.Y. Wang, L. Li, N.L. Liu, F. Xu, X.B. Wang, C.Z. Peng, J.W. Pan, Nature, 589, 214 (2021). DOI: 10.1038/s41586-020-03093-8

[3] C.J. Pugh, S. Kaiser, J.P. Bourgoin, J. Jin, N. Sultana, S. Agne, E. Anisimova, V. Makarov, E. Choi, B.L. Higgins, T. Jennewein, Quant. Sci. Technol., 2 (2), 024009 (2017).

DOI: 10.1088/2058-9565/aa701f

[4] H.Y. Liu, X.H. Tian, C. Gu, P. Fan, X. Ni, R. Yang, J.N. Zhang, M. Hu, J. Guo, X. Cao, X. Hu, G. Zhao, Y.Q. Lu, Y.X. Gong, Z. Xie, S.N. Zhu, Nat. Sci. Rev., 7 (5), 921 (2020). DOI: $10.1093 /$ nsr/nwz227

[5] S.K. Liao, W.Q. Cai, W.Y. Liu, L. Zhang, Y. Li, J.G. Ren, J. Yin, Q. Shen, Y. Cao, Z.P Li, F.Z. Li, X.W. Chen, L.H. Sun, J.J. Jia, J.C. Wu, X.J. Jiang, J.F. Wang, Y.M. Huang, Q. Wang, Y.L. Zhou, L. Deng, T. Xi, L. Ma, T. Hu, Q. Zhang, Y.A. Chen, N.L. Liu, X.B. Wang, Z.C. Zhu, C.Y. Lu, R. Shu, C.Z. Peng, J.Y. Wang, J.W. Pan, Nature, 549, 43 (2017). DOI: $10.1038 /$ nature 23655

[6] J. Yin, Y.H. Li, S.K. Liao, M. Yang, Y. Cao, L. Zhang, J.G. Ren, W.Q. Cai, W.Y Liu, S.L. Li, R. Shu, Y.M. Huang, L. Deng, L. Li, Q. Zhang, N.L. Liu, Y.A. Chen, C.Y. Lu, X.B. Wang, F. Xu, J.Y. Wang, C.Z. Peng, A.K. Ekert, J.W. Pan, Nature, 582, 501 (2020). DOI: 10.1038/s41586-020-2401-y

[7] V.L. Kurochkin, A.V. Khmelev, I.V. Petrov, A.V. Miller, A.A. Feimov, V.F. Mayboroda, M.Y. Balanov, V.V. Krushinsky, A.A. Popov, Y.V. Kurochkin, J. Phys.: Conf. Ser., 1680 (1), 012031 (2020). DOI: 10.1088/1742-6596/1680/1/012031 